\title{
Seroprevalence of major blood-borne infections among blood donors at Felege Hiwot referral hospital, northwest Ethiopia
}

\author{
Azene Dessie $^{1}$, Bayeh Abera ${ }^{2}$, Fisseha Wale ${ }^{2}$
}

\begin{abstract}
A cross-sectional study was conducted to determine the seroprevalence of major transfusion-transmissible infections among blood donors at Felege Hiwot referral hospital, northwest Ethiopia. The overall seroprevalence of major bloodborne pathogens among blood donors was 43.2\%, of which HBsAg, anti-HCV IgG, HIV seropositivity and syphilis accounted for $25 \%, 13.3 \%, 11.7 \%$ and $1.2 \%$ respectively. Crude seroprevalence of major blood born pathogens was significantly higher in commercial blood donors (56.6\%) compared to voluntary (17.6\%) and replacement blood donors (53.6\%) ( $\mathrm{p}=0.0001)$. [Ethiop.J.Health Dev. 2007;21(1):68-69]
\end{abstract}

\section{Introduction}

Although blood transfusion is one of the known therapeutic interventions that cut across a number of clinical disciplines, the practice is not without risks. In Africa, about 10-15 \% of HIV transmission had been related to blood transfusions (1) .The highest risk groups are children suffering from malaria and anaemia; women with pregnancy- related haemorrhage and victims of major trauma.

Felege Hiowt Referral Hospital is a 245 bed hospital located in Bahir Dar city, northwest Ethiopia. Being situated in a malaria dominant area, blood transfusion is one of the routine services in this hospital. However, most of the donated blood is screened for HIV alone. The national HIV prevalence among blood donors is $4.7 \%$ ( 6). However, there is paucity of information about the true burden of major transfusion-transmissible infections in the study area. The purpose of this study is, therefore, to determine the seroprevalence of common blood-borne infections among the donor population.

\section{Methods \\ A cross-sectional study was conducted at Felege Hiwot Referral Hospital and Bahir Dar Regional Health Research Laboratory from February 2006 to May 2006. Exclusion criteria for blood donation were current history of medication, recent history of operation, serious illness, previous blood transfusion, weight $<50 \mathrm{~kg}$, pregnant and lactating women, age $<18$ and $>60$ years and blood donation for 3 months prior to the current study. Applying these criteria, 324 apparently healthy blood donors who donated blood were included in the study. At the time of blood collection, socio-demographic variables of the study population were collected using a structured questionnaire. In this study, commercial donor is a donor who gives blood for money or other form of payment and a replacement donor is a donor who gives blood when it is required by a member of the patient's family.}

Serological assays for HIV infection, Hepatitis B virus, Hepatitis $C$ virus and Syphilis were screened by using $4^{\text {th }}$ generation ELISA, Vironostika HIV Uni-Form II AG/Ab (Bio-Merieux, Boxtel, Netherlands), an immunoassay

ELISA Hepanostika HBSAg UNi-Form II (Bio-Merieux, Boxtel, Netherlands), using the Human anti-HCV $3^{\text {rd }}$ generation ELISA (Human Gasellschaft for Bio-chemical and diagnostic $\mathrm{MbH}$, Germany) and using Rapid Plasma Reagnic (BHAT-BIO-TECH, Banglor, India) respectively. Pearson Chi-Square and 95\% confidence interval were employed for group comparison of blood donors and association of variables.

\section{Results}

A total of 324 blood donors including 283 males and 41 females donated blood during the study period, out of which 152 (46.9\%), 138 (42.6\% ) and 34 (10.5\% ) were commercial, replacement and voluntary donors respectively. The overall seroprevalence of major bloodborne pathogens (HIV, HBV, HCV and syphilis) among blood donors was $43.2 \%$. Seroprevalence of hepatitis B virus was the highest (25\%) followed by hepatitis $C$ virus (13.3\%), human immunodeficiency virus (11.7\%) and syphilis (1.2\%) (Table: 1$)$. The crude seroprevalence of major blood born pathogens was significantly higher in commercial (56.6\%) blood donors compared to voluntary (17.6\%) and replacement blood donors (53.6\%) at $\mathrm{p}=0.001)$.

Among multiple infected blood donors, HIV seropositive blood donors (31.6\%) were found to be significantly coinfected with HBV, HCV and syphilis compared with HIV seronegative blood donors (4.9\%) at $\mathrm{P}=0.0001$. Among the co-infections HBV and HCV infections were found to be significantly co-existing in our study population $(\mathrm{p}<0.05)$.

\footnotetext{
${ }^{1}$ Department of Paediatrics and Child Health, Felege Hiwot Referral Hospital, P.O.Box 891, Bahir Dar, Ethiopia, Tel: 251918 763686, Fax: 251582 220917, E-mail: admengistu2000@yahoo.com; ${ }^{2}$ Medical Microbiologist, Bahir Dar Regional Health Research Laboratory, P.O.Box 641; Bahir Dar,Ethiopia
} 


\begin{tabular}{|c|c|c|}
\hline $\begin{array}{l}\text { Transfusion transmissible } \\
\text { Infections (TT/s) }\end{array}$ & Number & Percent \\
\hline $\mathrm{HBV}$ & 81 & 25 \\
\hline $\mathrm{HCV}$ & 43 & 13.3 \\
\hline HIV & 38 & 11.7 \\
\hline Syphilis & 4 & 1.2 \\
\hline None of the screened TTIs & 184 & 56.8 \\
\hline Total & 324 & 100.00 \\
\hline
\end{tabular}

\section{Discussion}

This study demonstrated that the seroprevalence of blood-borne infections in the donor population is unacceptably high (43.2\%).This finding is by and large higher than similar reports from other developing settings. Occurrence of at least one pathogen among blood donors was reported to be $15.9 \%$ and $26.2 \%$ from studies in Tanzania and Cameroon respectively $(2,3)$. Hence, even when compared with other resourceconstrained settings, the burden of transfusiontransmissible infections is quite high in our study population.

As with a case of previous report from South Africa (4), the prevalence of HIV infection in the blood donor population was found to be high (11.7\%). Nearly a decade ago, Rahlenbeck SI et al (7) reported a $16.7 \%$ prevalence of HIV infection among Ethiopian blood donors. Another local research work from Gondar showed crude seroprevalence of HIV was $10.6 \%$ in men and $11.9 \%$ in women among a similar study population which is in parallel with our finding (8).

Consistent with the findings of this study, the prevalence of HBV infection was reported to be higher than that of HCV infection in another East African study (5). We found that $8 \%$ of the overall blood donors and $18.6 \%$ of the infected persons had co-infections. One of the other important findings we observed in this study was the statistically significant occurrence of dual blood-born infections among seropositive donors with HIV infection than the scenario in HIV seronegative ones. Such a higher prevalence of co-infections among HIV infected persons is likely to have a clear implication in the routine clinical care of HIV infected patients.

In agreement with the findings of this study, other research work also demonstrated high infection burden among commercial blood donors while voluntary donors were found to be comparatively safe (2).In this research work, more than half (56.6\%) of the blood donors were commercial, which is one of the clear explanations for the unacceptably high prevalence of transfusiontransmissible infections. In conclusion, blood safety is an issue of major concern at Felege Hiwot Referral Hospital. Hence, blood donations should be routinely screened for HIV, HBV, HCV and syphilis using standard methods. A strict selection criterion of donors, with emphasis on getting voluntary donors is also highly recommended.

\section{Acknowledgment}

We are grateful to the Amhara Region HIV/AIDS prevention and control office for financing the research work. We also thank Bahir Dar Regional Health Research Laboratory for supply of the materials during the study.

\section{References}

1. Fleming AF. HIV and blood transfusion in subSaharan Africa. Transfus sci.1997;18(2):167-79.

2. Matee MI, Magesa PM, Lyamuya EF. Seroprevalence of human immunodeficiency virus, hepatitis B and C viruses and syphilis infections among blood donors at the Muhimbili National Hospital in Dare Es Salam, Tanzania. BMC Public Health.2006;6(1):21-25.

3. Mbanya DN, Takam D, Ndumbe PM. Serological findings amongst first-time blood donors in Yaounde, Cameroon: Is safe donation a reality or a myth? Transfus Med 2003;13(5):267-73.

4. Hens AP, Benjamin RJ, Swanevelder JP, Laycock ME, Pappalardo BL, Crookes RL, Wright DJ, Bush MP. Prevalence of HIV-1 in blood donations following implementation of a structured blood safety policy in South Africa. JAMA 2006;295(5): 519-26.

5. Matee MI, Lyamuya EF, Mbena EC, Magessa PM, Sufi J,Marwa GJ et al. Prevalence of transfusionassociated viral infections and syphilis among blood donors at Muhimbii Medical Centre, Dares Salaam, Tanzania, East Afr Med J. 1999;76(3):167-71.

6. Federal Ministry of Health. Disease Prevention and Control Department. AIDS in Ethipoia, 5th Report. Addis Ababa.

7. Rahlenbeck SI, Yohannes G, Molla K, Reifen R, Assefa A. Infection with HIV, syphilis and hepatitis B in Ethiopia: a survey in blood donors. Int J STD AIDS. 1997;8(4):261-4.

8. Assefa A, Rahlenbeck S, Molla K, Alemu S.Seroprevalence of HIV-1 and syphilis antibodies in Gondar, Ethiopia, 1989-1993. Acquir Immune Defic Syndr.1994;7(12):1282-5. 